\title{
Restoration of Error-Diffused Images Using Projection Onto Convex Sets
}

\author{
Gozde Bozkurt Unal, Student Member, IEEE, and A. Enis Çetin, Senior Member, IEEE
}

\begin{abstract}
In this paper, a novel inverse halftoning method is proposed to restore a continuous tone image from a given half-tone image. A set theoretic formulation is used where three sets are defined using the prior information about the problem. A new spacedomain projection is introduced assuming the halftoning is performed using error diffusion, and the error diffusion filter kernel is known. The space-domain, frequency-domain, and space-scale domain projections are used alternately to obtain a feasible solution for the inverse halftoning problem which does not have a unique solution.
\end{abstract}

Index Terms-Error diffusion, inverse error diffusion, inverse halftoning, projection onto convex sets (POCS), restoration.

\section{INTRODUCTION}

I NVERSE halftoning is the problem of recovering a continuous tone (contone) image from a given half-tone image. Contone images are needed in many practical applications. However, inverse halftoning problem is ill-posed because halftoning is a many-to-one mapping, and does not a have a unique solution [1]. Therefore, incorporation of all available information significantly improves the quality of the solution and this leads to the fact that a set theoretic formulation is ideally suitable for the inverse halftoning problem that has many feasible solutions.

The previous inverse halftoning methods employ space-domain operations, frequency-domain operations, or both, or only space-scale domain operations [1]-[8]. The simplest approach is low-pass filtering the half-tone image to remove the high-frequency components where the halftoning noise is mostly concentrated. Different low-pass filters have been used, such as half-band low-pass in [1], Gaussian low-pass, and low-pass filtering based on singular value decomposition (SVD) [2]. However, low-pass filtering alone does not work well as this also destroys high-frequency information of the original image.

A projection algorithm, based on the maximum a posteriori probability (MAP) projection is proposed in [1]. A similar MAP estimation method is also proposed in [3] where a constrained optimization is solved using iterative techniques.

Xiong et al. [4] proposed an inverse halftoning scheme using wavelets. The idea behind the wavelet decomposition of

\footnotetext{
Manuscript received November 10, 1999; revised September 1, 2001. This work was supported in part by NATO under Grant CRG-971117 and was carried out at Bilkent University, Ankara, Turkey. The associate editor coordinating the review of this manuscript and approving it for publication was Prof. Timothy J. Schulz.

G. B. Unal is with the Electrical and Computer Engineering Department, North Carolina State University, Raleigh, NC 27695-7914 USA.

A. E. Çetin is with Sabanci University, Istanbul, Turkey, on leave from the ECE Department, Bilkent University, Ankara 06533, Turkey.

Publisher Item Identifier S 1057-7149(01)10569-5.
}

a half-tone image is to selectively choose useful information from each subband. This approach is a space-scale domain method and no prior knowledge about the halftoning process is assumed.

The method of projection onto convex sets (POCS) is used in [5], [2], [6], where information known about the problem is expressed in the form of two constraint sets. In [2], [5], and [6], the halftoning process is assumed to be known a priori. Based on this information and the smoothness of most natural images, convex sets are defined. The iterative restoration algorithm is developed by making successive projections onto the convex sets. The first set $C_{1}$ is the set of all contone images when halftoned produce the observed half-tone image, and the second is the set of all images bandlimited to a certain low-pass band. The computational cost of the space-domain projection in [2] turns out to be very high.

In this paper, we define a new family of sets $C_{1, n}$, representing the space-domain information. It turns out that the intersection of the sets $C_{1, n}$, is the set $C_{1}$ originally defined in [2]. The use of the sets $C_{1, n}$, leads to a computationally more efficient reconstruction algorithm because, in each iteration of the POCS algorithm we do not update the entire image as in [2] but we modify only the pixels that do not meet the requirements. We also take advantage of the frequency, and space-scale (or wavelet) domain projections which represent the prior knowledge about the error diffusion filter kernel, and the relatively smooth character of the natural images.

The theory and simulation results are presented in Sections II and III, respectively, and it is experimentally observed that higher quality images can be obtained compared to [2], [1], [4].

\section{A SET TheOretic InVERSE HALFTONING}

The block diagram of an error diffusion encoder is given in Fig. 1. The inverse halftoning problem can be stated as follows. Given the half-toned image $\boldsymbol{y}=y\left(n_{1}, n_{2}\right)$ and the two-dimensional (2-D) finite-impulse response (FIR) error diffusion filter kernel $\boldsymbol{h}$, estimate the original image $\boldsymbol{x}=x\left(n_{1}, n_{2}\right)$. In this section, we first introduce the POCS-based framework and then define the space, frequency, and the space-scale sets that is used in the reconstruction algorithm.

Let $x[n]$ and $y[n]$ be the $n$th pixels of the $N_{1} \times N_{2}$ images $\boldsymbol{x}$ and $\boldsymbol{y}$, respectively [in error diffusion, images are scanned rowwise or colomnwise therefore we also represent images using a single index $\left.n=\left(n_{1}-1\right) \times N_{2}+n_{2}\right]$. Corresponding to each observed pixel, $y[n]$, a space-domain set $C_{1, n}, n=1,2, \ldots, L=$ $N_{1} \times N_{2}$ (number of pixels) is defined as follows. The set $C_{1, n}$ contains all contone images that result in the observed error diffused pixel $y[n]$ at index $n$. The intersection of these sets 


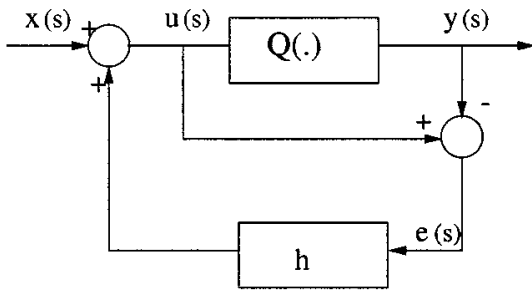

Fig. 1. Block diagram of error diffusion method.

\begin{tabular}{|l|l|l|}
\hline 0 & 0 & $7 / 16$ \\
\hline $3 / 16$ & $5 / 16$ & $1 / 16$ \\
\hline \multicolumn{3}{|c}{$h$}
\end{tabular}

\begin{tabular}{|c|c|c|}
\hline 0 & 1 & $-7 / 16$ \\
\hline$-3 / 16$ & $-5 / 16$ & $-1 / 16$ \\
\hline \multicolumn{3}{|c}{ I-h }
\end{tabular}

Fig. 2. Kernels for the filters $\mathbf{h}$ and $\mathbf{I}-\mathbf{h}$.

$C_{1}=\bigcap_{n} C_{1, n}$ is the set of all contone images $\boldsymbol{x}$ producing the observed error diffused image $\boldsymbol{y}$. Clearly, the sets $C_{1, n}$ are much larger than the set $C_{1}$ and they all contain $C_{1}$ because any image in $C_{1}$ produces the individual pixel $y[n]$ after error diffusion thus it is also in $C_{1, n}$. As $n$ goes from 1 to $\mathrm{L}$, all the pixels of $\boldsymbol{y}$ are covered by the family of sets $C_{1, n}$, and the set $C_{1}$ is formed by the intersection.

The set $C_{1}$ which is used in [6] and [2] or the sets $C_{1, n}$ are too large to determine the solution of the reconstruction problem. One has to assume other constraints on the original image to estimate the original image. Most natural images are of low-pass in nature whereas the half-toned image contains a lot of high-frequency components. Therefore one can assume that the original image is a member of a set $C_{2}$ whose members are bandlimited images by $\omega_{o}$ in both directions. Even if the original image is not a strictly bandlimited image most of its energy is concentrated in low frequencies. The errors made in pixel values by projecting a current iterate onto the set $C_{2}$ can be corrected by subsequent projections onto the sets $C_{1, n}$.

The projection onto the set $C_{2}$ is equivalent to ideal low-pass filtering which is simply implemented by an FIR low-pass filter. The choice of a small $\omega_{0}$ may blur the image. On the other hand a large $\omega_{0}$ may not be effective.

In order to preserve the sharpness of the image, the wavelet transform (WT) extrema information can be used. WT extrema occur at the edge locations which can be easily estimated from the half-toned image as the significant edges are clearly visible in a typical half-toned image. Therefore the set $C_{3}$ of images having the same significant WT local extrema as the original image is a good choice to provide sharpness to the low-pass filtered image. The set $C_{3}$ is shown to be convex in [7]-[10].

The POCS-based iterative algorithm starts with an initial estimate $\boldsymbol{x}_{0}$, which is successively projected onto the sets $C_{1, n}$, $C_{2}$, and $C_{3}$ as follows:

$$
\begin{array}{r}
\boldsymbol{x}_{\ell+1}=\left(P_{1,0} \circ \ldots \circ P_{1, L} \circ P_{2} \circ P_{3}\right) \boldsymbol{x}_{\ell}, \\
\qquad=0,1,2, \ldots
\end{array}
$$

where $P_{1, n}$ represents the spatial projection which is described in the next subsection, $P_{2}$ represents low-pass filtering which is the frequency-domain projection, and $P_{3}$ represents the wavelet-domain space-scale projection which can be implemented using the algorithm described in [8]. All three projections, or any two can be used alternately. The algorithm is globally convergent to a solution which is in the intersection of all the convex sets regardless of the initial estimate, and the order of the projections is immaterial [11]. The iterations are stopped when the difference between the images at successive iterations become insignificant.

In our iterative algorithm summarized by (1), a projection cycle is completed by performing orthogonal projections onto the sets $C_{1, n}$, as the original image is clearly a member of them.

In our inverse halftoning method, we also use nonorthogonal projections during the reconstruction process. As discussed in [12] and [13], nonorthogonal projections may not only improve the speed of convergence but also improve the quality of the reconstructed image. Orthogonal projections onto a convex set yields an image at the boundary of the set. On the other hand, by performing a nonorthogonal projection onto a convex set an image inside the set is obtained.

\section{A. Projection Onto the Sets $\boldsymbol{C}_{1, n}$}

Consider the error diffusion system shown in Fig. 1. Let $\boldsymbol{u}$ be the image before the quantizer $\boldsymbol{Q}($.$) . The error image \boldsymbol{e}=\boldsymbol{u}-\boldsymbol{y}$ and $\boldsymbol{u}=\boldsymbol{x}+\boldsymbol{h} * \boldsymbol{e}$ where $*$ represents the 2-D convolution. The image $\boldsymbol{u}$ can be expressed in terms of the original image $\boldsymbol{x}$, and the output image $\boldsymbol{y}$ as follows:

$$
u=(\delta-h)^{-1} *(\boldsymbol{x}-h * \boldsymbol{y})
$$

where $\delta$ is the 2-D unit sample sequence. The kernels for the FIR filters $\boldsymbol{h}$ and $\boldsymbol{\delta}-\boldsymbol{h}$ are given in Fig. 2.

The set $C_{1, n}$ is based on the observation that for a given output pixel $y[n]$, the upper and lower bounds on the pixel $u[n]$ can be determined according to the quantization levels. For example, if $y[n]=0$ then $0 \leq u[n] \leq 127$ for 1-bit error diffusion with a uniform quantizer. The sets $C_{1, n}$ can then be defined as follows:

$$
\begin{array}{r}
C_{1, n}=\left\{x: d_{l o, n} \leq(\boldsymbol{\delta}-\boldsymbol{h})^{-1} *(\boldsymbol{x}-\boldsymbol{h} * \boldsymbol{y})[n] \leq d_{u p, n}\right\} \\
n=1,2, \ldots, L
\end{array}
$$

where $d_{l o, n}$ and $d_{u p, n}$ are, respectively, the lower and upper bounds for the nth pixel $n=\left(n_{1}-1\right) \times N_{2}+n_{2}$. Since the convolution operation is linear, the set $C_{1, n}$ is essentially a hyperslab (a region bounded by two parallel hyperplanes) and it is, therefore, a closed and convex set in $\mathbf{R}^{L}$. For convenience, we define $\overline{\boldsymbol{x}}=\boldsymbol{x}-\boldsymbol{h} * \boldsymbol{y}$. Since $\boldsymbol{h}$ and $\boldsymbol{y}$ are known, one can easily obtain $\boldsymbol{x}$ from $\overline{\boldsymbol{x}}$ or vice versa. Let us represent $\boldsymbol{u}$ in terms of $\overline{\boldsymbol{x}}$ as $\boldsymbol{u}=\boldsymbol{w} * \overline{\boldsymbol{x}}$ where the 2-D IIR inverse filter $\boldsymbol{w}=(\boldsymbol{\delta}-\boldsymbol{h})^{-1}$ can be approximated by a causal 2-D FIR filter using a method described in [14] for inverse filtering for image restoration. The pixel $\boldsymbol{u}[n]=(\boldsymbol{w} * \overline{\boldsymbol{x}})[n]$ can be expressed as $u\left(n_{1}, n_{2}\right)=\sum_{k_{1}, k_{2} \in F_{w}} \omega\left(k_{1}, k_{2}\right) \bar{x}\left(n_{1}-k_{1}, n_{2}-k_{2}\right)$, where $\omega\left(n_{1}, n_{2}\right)$ are the coefficients of the filter $w$, and $F_{w}$ is the corresponding causal support region. Since the above equation is a 


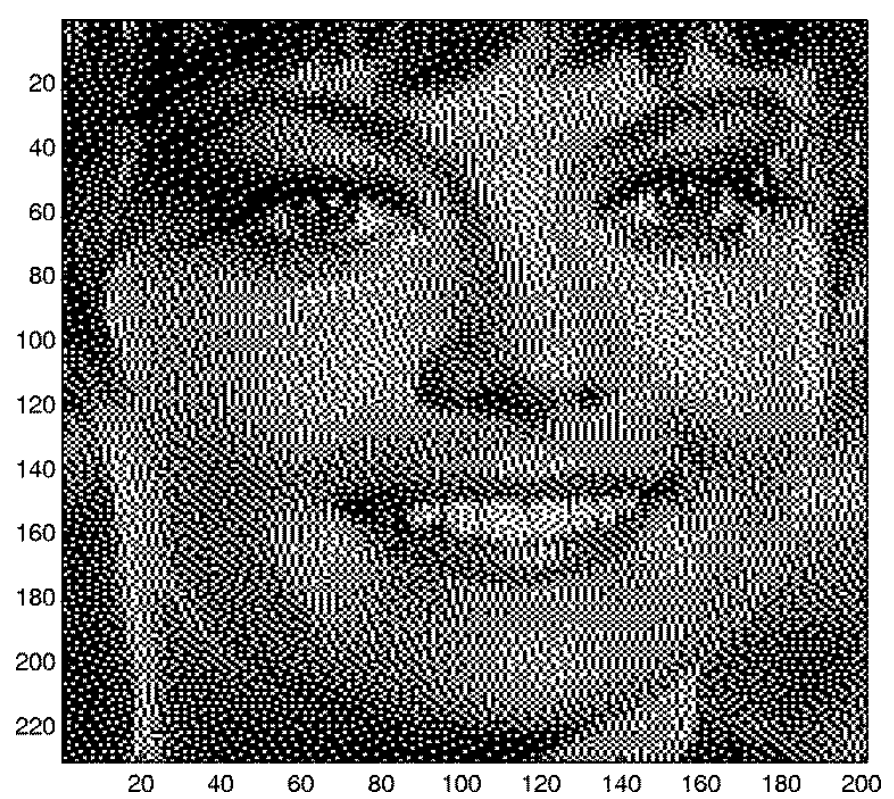

Fig. 3. Zelda image error-diffused to $1 \mathrm{bpp}$.

2-D convolution operation, it is a hyperplane in $R^{L}$. The constraints on $u\left(n_{1}, n_{2}\right)$ given in (3) can be rewritten as

$$
d_{l o, n} \leq \sum_{k_{1}, k_{2} \in F_{w}} \omega\left(k_{1}, k_{2}\right) \bar{x}\left(n_{1}-k_{1}, n_{2}-k_{2}\right) \leq d_{u p, n}
$$

which is a hyperslab (a region bounded by two parallel hyperplanes), and therefore it is a closed and convex set in $\boldsymbol{R}^{L}$. Since the upper and lower bounds $d_{u p, n}$ and $d_{l o, n}$, and the kernel $\omega\left(k_{1}, k_{2}\right)$ are known, the projection onto the set $C_{1, n}$ can be obtained as follows. Let $\overline{\boldsymbol{x}}_{p}$ be the current iterate. The next iterate $\overline{\boldsymbol{x}}_{p+1}$ is obtained by solving the optimization problem

$$
\min \left\|\overline{\boldsymbol{x}}_{p+1}-\overline{\boldsymbol{x}}_{p}\right\|^{2} \text { subject to (4). }
$$

If $\overline{\boldsymbol{x}}_{p}$ satisfies the constraints (4), then $\overline{\boldsymbol{x}}_{p+1}=\overline{\boldsymbol{x}}_{p}$. Otherwise

$$
\begin{aligned}
\bar{x}_{p+1}\left(l_{1}, l_{2}\right)= & \bar{x}_{p}\left(l_{1}, l_{2}\right)+\lambda \\
& \times\left(\frac{d_{q}-\sum_{k_{1}, k_{2}} \omega\left(k_{1}, k_{2}\right) \bar{x}_{p}\left(n_{1}-k_{1}, n_{2}-k_{2}\right)}{\sum_{k_{1}, k_{2}} \omega\left(k_{1}, k_{2}\right)^{2}}\right) \\
& \times w\left(n_{1}-l_{1}, n_{2}-l_{2}\right)
\end{aligned}
$$

where $l_{1}=1,2, \ldots, N_{1} ; l_{2}=1,2, \ldots, N_{2}$ and $d_{q}$ is either $d_{l o, n}$, if $\left(\boldsymbol{\omega} * \overline{\boldsymbol{x}}_{p}\right)[n]<d_{l o, n}$ or $d_{q}=d_{u p, n}$, if $\left(\boldsymbol{\omega} * \overline{\boldsymbol{x}}_{p}\right)[n]>$ $d_{u p, n}$, and $\lambda$ is a relaxation parameter, and if it remains between zero and two, the convergence of the POCS procedure is assured [11]. If $\lambda=1$, then the projection is an orthogonal projection. When $0<\lambda<1$, an image inside the set $C_{1, n}$ is obtained without effecting the convergence process. In fact, the use of nonorthogonal projections improves the speed of convergence and the quality of the reconstructed image.

The projection operation described in (6) is a local operation around the pixel $x_{p}[n]=x\left(n_{1}, n_{2}\right)$ (or equivalently $\bar{x}_{p}[n]=$ $\bar{x}_{p}\left(n_{1}, n_{2}\right)$ ), because the filter $\boldsymbol{w}$ is only nonzero in the support region $F_{w}$, whereas the pixel range is from $n=1$ to $n=L$.
The projection given in (6) is performed pixel by pixel involving the block defined by the causal support region $F_{w}$. During each iteration, only the pixels defined by $F_{w}$ are updated.

Equation (6) is essentially the projection onto one of the hyperplanes bounding the set $C_{1, n}$. Once an estimate for the image $\overline{\boldsymbol{x}}$ is obtained, an estimate for $\boldsymbol{x}$ is obtained from $\boldsymbol{x}=\overline{\boldsymbol{x}}+\boldsymbol{h} * \boldsymbol{y}$.

This scheme can be easily extended to the case of multilevel error-diffusion in which the quantizer is not binary. In the binary case, the bounds $d_{l o, n}$ and $d_{u p, n}$ can take only, -128 and 255,128 and $255+128$, respectively, for 8 bit/pel images. In the multilevel case, the bounds are simply determined according to the quantization levels.

This space-domain projection is different from the space-domain projection described in [2] in two aspects:

1) the convex sets that we define are different from the set $C_{1}$, defined in [2];

2) our method is developed for the widely used Floyd-Steinberg error diffusion method, whereas [2] is developed for a sigma-delta type error diffusion algorithm.

Due to the nature of our convex sets $C_{1, n}$, the projection operation described in (6) is very simple to implement. This leads to a computationally more efficient reconstruction algorithm because, in each iteration of the POCS algorithm, we do not update the entire image as in [2], but we modify only the pixels that do not meet the requirements.

The computational cost of the projections onto the sets $C_{1, n}$ described in (6) is comparable to the projection operation performed in another domain in [6]. Both projections are essentially based on the convolution operation. The support region $F_{w}$ in (6) is larger than the support of the filter used in [6]. On the other hand, filtering is performed over the entire image in [6], whereas we update only the pixels that do not meet the requirements.

\section{B. Frequency-Domain Projection}

An important property of most natural images is smoothness compared to artificial images. This information can be imposed into the restoration process in the form of low-pass filtering. Therefore, the frequency-domain projection onto the set $C_{2}$ consists of bandlimiting the observed signal in some way. The simplest approach is to low-pass filter the image in order to remove the high-frequency content which contains halftoning noise. For the frequency-domain projection, we either use a simple Gaussian low-pass filter $g\left(n_{1}, n_{2}\right)=K e^{-\left(n_{1}^{2}+n_{2}^{2} / 2 \sigma^{2}\right)}$, for $-3 \leq n_{1}, n_{2} \leq 3$, where $K$ is a scaling factor used to make the dc gain of the filter unity. The $\sigma^{2}$ controls the bandwidth of the low-pass filter; or we use low-pass filters with passbands of $[-\pi / 2, \pi / 2] \times[-\pi / 2, \pi / 2],[-2 \pi / 3,2 \pi / 3] \times[-2 \pi / 3,2 \pi / 3]$, or $[-3 \pi / 4,3 \pi / 4] \times[-3 \pi / 4,3 \pi / 4]$.

\section{Space-Scale Domain Projection}

The edges in an image produce local WT extrema in the space-scale domain in wavelet subimages (or in high-low, lowhigh, and high-high subimages) [7]. It is proved that the wavelet extrema information corresponds to convex sets in the set of square summable images $\ell_{2}$ [7]-[9]. Therefore, the edge information can be used in the reconstruction algorithm by properly defining a set corresponding to the significant local extrema 
in the wavelet domain. Let the set $\boldsymbol{C}_{3}$, contain all the images having the same significant WT local extrema as the original image. The key idea is to estimate the edges of the original image from the half-toned image by selecting the significant WT extrema of the half-toned image, and the restored image is forced to have the same extrema in the wavelet space-scale domain. This provides sharpness to the restored image by protecting the significant high-frequency components of the image, whereas a simple low-pass filtering characterized by set $C_{2}$ will smooth out all of the sharp edges of the original image.

In a typical half-toned or dithered image, edges of the original image are clearly visible. In order to highlight the significant edges, one can manually mark them. Let us assume that $x_{\text {wav }}\left(n_{o}, m_{o}\right)$ is a local maximum in the wavelet domain. It can be expressed as

$$
\begin{aligned}
x_{\text {wav }}\left(n_{o}, m_{o}\right)=\sum_{k_{1}, k_{2}} x & \left(k_{1}, k_{2}\right) h_{\text {wav }} \\
& \times\left(2 \times n_{o}-k_{1}, 2 \times m_{o}-k_{2}\right)
\end{aligned}
$$

where $\boldsymbol{x}$ is the original image and $\boldsymbol{h}_{w a v}$ is a 2-D filter used in the filterbank structure implementing the WT. If oversampled, WT is used then the above equation becomes

$$
x_{w a v}\left(n_{o}, m_{o}\right)=\sum_{k_{1}, k_{2}} x\left(k_{1}, k_{2}\right) h_{w a v}\left(n_{o}-k_{1}, m_{o}-k_{2}\right)
$$

which is simply the 2-D convolution evaluated at $\left(n_{o}, m_{o}\right)$ location. This is a convex set in $\ell_{2} \times \ell_{2}$ (the set of 2-D square summable sequences), and it also corresponds to a convex set in $R^{L}$.

The projection onto this set can be carried out as follows [8]:

$$
\begin{aligned}
& x_{p+1}\left(l_{1}, l_{2}\right) \\
& =x_{p}\left(l_{1}, l_{2}\right)+\lambda \\
& \times\left(\frac{x_{w a v}\left(n_{o}, m_{o}\right)-\sum_{k_{1}, k_{2}} h_{w a v}\left(k_{1}, k_{2}\right) x_{p}\left(n_{o}-k_{1}, m_{o}-k_{2}\right)}{\sum_{k_{1}, k_{2}} h_{w a v}\left(k_{1}, k_{2}\right)^{2}}\right) \\
& \times h_{w a v}\left(n_{o}-l_{1}, m_{o}-l_{2}\right)
\end{aligned}
$$

where $l_{1}=1,2, \ldots, N_{1} ; l_{2}=1,2, \ldots, N_{2} ; \boldsymbol{x}_{p}$ is the current iterate; and $x_{p+1}$ is the projection. In (9), $\lambda=1$ corresponds to the orthogonal projection. In order to implement the above iteration the value of the extremum $x_{w a v}\left(n_{o}, m_{o}\right)$ must be known. This may not be exactly available in a typical dithering problem. But this problem can be overcome as follows. Since $x_{w a v}\left(n_{o}, m_{o}\right)$ is a local maximum $x_{\text {wav }}\left(n_{o}+\right.$ $\left.1, m_{o}\right) \leq x_{w a v}\left(n_{o}, m_{o}\right)$, and this means that

$$
\begin{aligned}
& \sum_{k_{1}, k_{2}} x\left(k_{1}, k_{2}\right) h_{w a v}\left(n_{o}+1-k_{1}, m_{o}-k_{2}\right) \\
& \leq \sum_{k_{1}, k_{2}} x\left(k_{1}, k_{2}\right) h_{w a v}\left(n_{o}-k_{1}, m_{o}-k_{2}\right)
\end{aligned}
$$

or

$$
\begin{aligned}
& \sum_{k_{1}, k_{2}} x\left(k_{1}, k_{2}\right)\left(h_{\text {wav }}\right.\left(n_{o}+1-k_{1}, m_{o}-k_{2}\right) \\
&\left.-h_{w a v}\left(n_{o}-k_{1}, m_{o}-k_{2}\right)\right) \leq 0
\end{aligned}
$$

which is a half-space in $R^{L}$. Therefore, it corresponds to a closed and convex set. The projection onto this set can be carried out as follows. Let $\boldsymbol{x}_{p}$ be the current iterate. If $\sum_{k_{1}, k_{2}} x_{p}\left(k_{1}, k_{2}\right)\left(h_{w a v}\left(n_{o}+1-k_{1}, m_{o}-k_{2}\right)-h_{w a v}\left(n_{o}-\right.\right.$ $\left.\left.k_{1}, m_{o}-k_{2}\right)\right) \leq 0$ then $\boldsymbol{x}_{p+1}=\boldsymbol{x}_{p}$ otherwise

$$
\begin{aligned}
& x_{p+1}\left(l_{1}, l_{2}\right)=x_{p}\left(l_{1}, l_{2}\right) \\
&+\lambda\left(\frac{\sum_{k_{1}, k_{2}} h_{w d}\left(k_{1}, k_{2}\right) x_{p}\left(k_{1}, k_{2}\right)}{\sum_{k_{1}, k_{2}} h_{w d}\left(k_{1}, k_{2}\right)^{2}}\right) h_{w d}\left(l_{1}, l_{2}\right)
\end{aligned}
$$

where $l_{1}=1,2, \ldots, N_{1} ; l_{2}=1,2, \ldots, N_{2} ;$ and $h_{w d}\left(k_{1}, k_{2}\right)$ $=h_{w a v}\left(n_{o}-k_{1}, m_{o}-k_{2}\right)-h_{w a v}\left(n_{o}+1-k_{1}, m_{o}-k_{2}\right)$. Similarly, $x_{\text {wav }}\left(n_{o}, m_{o}+1\right) \leq x_{\text {wav }}\left(n_{o}, m_{o}\right)$. This defines another half-space with boundary $\sum_{k_{1}, k_{2}} x\left(k_{1}, k_{2}\right)\left(h_{\text {wav }}\left(n_{o}-\right.\right.$ $\left.\left.k_{1}, m_{o}+1-k_{2}\right)-h_{\text {wav }}\left(n_{o}-k_{1}, m_{o}-k_{2}\right)\right)=0$. The projection operation onto the above half-space can be carried out as in (12)

Another approach is to use the wavelet-based single step inverse halftoning method [4]. Although this method cannot be considered as an orthogonal projection due to the cross-scale correlation operation, it is relatively easy to implement and can be incorporated into the iterative restoration procedure. In [4], important high-frequency information describing the signal, particularly information in edge regions, are retained by choosing the WT extrema locations selectively from each subband resulting from the wavelet decomposition of the half-toned image. In our iterative restoration algorithm, this method is used as an initial step in some of the simulation results.

\section{SimUlation Results}

To demonstrate the performance of our POCS-based inverse halftoning method, we present simulation results using $512 \times 512$ Peppers and Zelda images. We compare the new method with some state-of-the-art inverse halftoning techniques in terms of their PSNRs. In the first group of simulations, we use space-domain and frequency-domain projections alternately. The first estimate of the contone image is obtained by low-pass filtering the half-toned image with $g\left(n_{1}, n_{2}\right)$. Then we perform our spatial projections. After that, we again use low-pass filtering, and go on in an alternating fashion. A section of the original $8 \mathrm{bpp}$ Zelda image error-diffused to $1 \mathrm{bpp}$ is shown in Fig. 3. After low-pass filtering, the resultant image with PSNR $=32.85 \mathrm{~dB}$ is shown in Fig. 4(a). This image is used as an initial estimate and after two sets of iterations, an image shown in Fig. 4(b) with PSNR $=33.45$ dB is obtained. The resulting image is quite sharp, and its visual quality is high. The details are restored while much of the halftoning noise existing in the first estimate is removed.

We compare our results with those in [2] in Table I for the Lena image. The PSNR improvement achieved by the proposed method is about $0.8 \mathrm{~dB}$ higher than the ones in [2], and the image quality is higher.

Apart from the binary error diffusion coding, we carried out simulation studies for images error-diffused to $2 \mathrm{bpp}$, as shown in Fig. 5 for the Zelda image. We use our method tailored for 


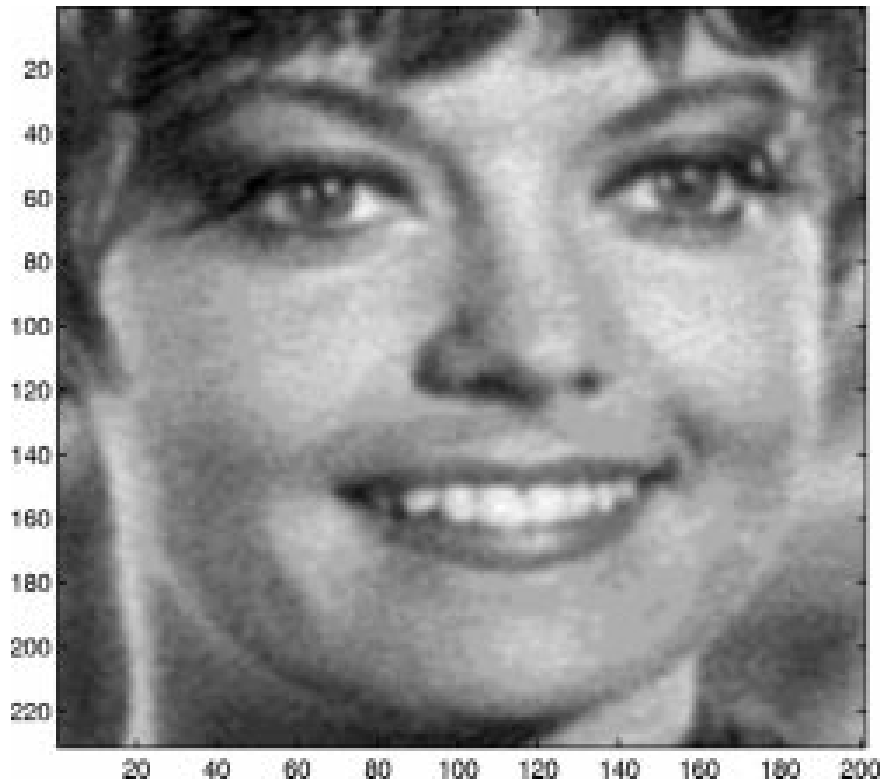

(a)

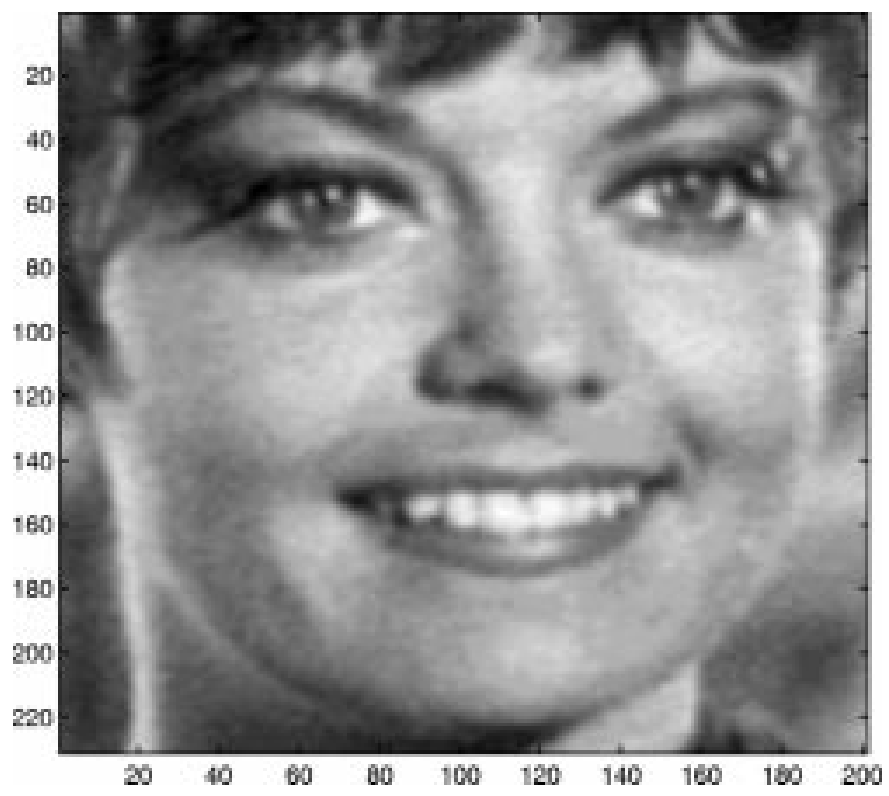

(b)

Fig. 4. (a) First estimate (PSNR $=32.85 \mathrm{~dB}$ ). (b) Restored Zelda image $(\mathrm{PSNR}=33.45 \mathrm{~dB})$

TABLE I

COMPARISON OF PSNRs (dB) FOR THE INVERSE HALF-TONING METHODS IN [2], AND OUR METHOD FOR THE LENA IMAGE. GLPF, LPF, AND SVD DENOTE THE TYPE OF FREQUENCY-DOMAIN PROJECTION

\begin{tabular}{c|c|c}
\hline [2] (GLPF) & [2] (SVD) & Our Method (GLPF, LPF) \\
\hline \hline 29.4 & 30.4 & 31.23 \\
\hline
\end{tabular}

the multilevel case. The PSNR improvement over the initial estimate is about $0.6 \mathrm{~dB}$ with our POCS-based method after two sets of iterations, and our restoration results in a sharp and faithful reproduction, as can be seen in Fig. 5 (PSNR $=35.39 \mathrm{~dB}$ ).

We can use wavelet-based space-scale domain projection in [4] as the initial estimate in our method. Let $x_{w}$ be the restored

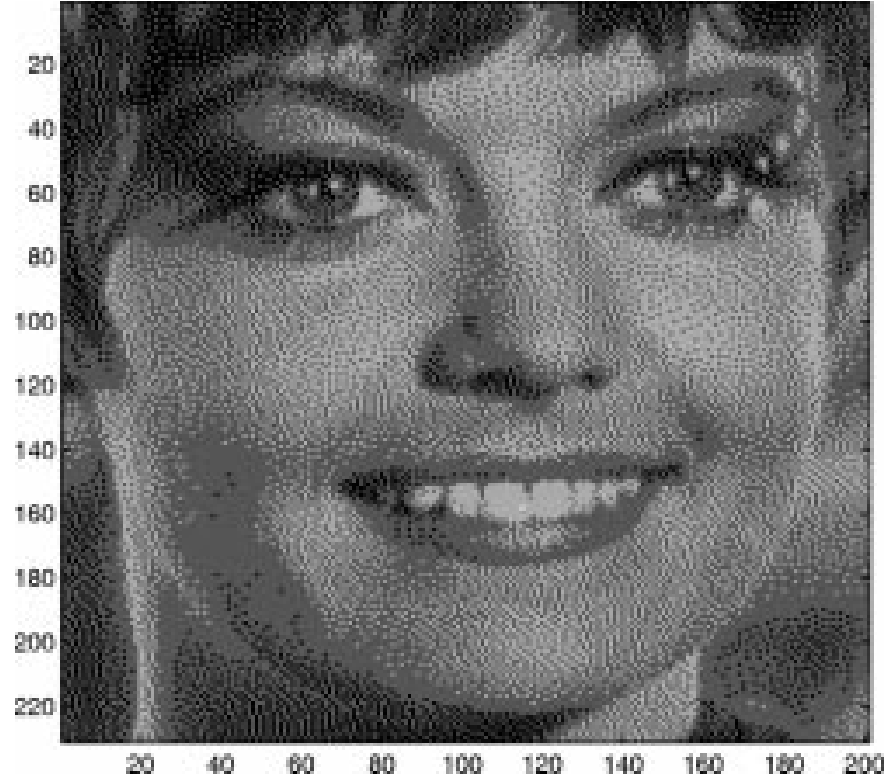

(a)

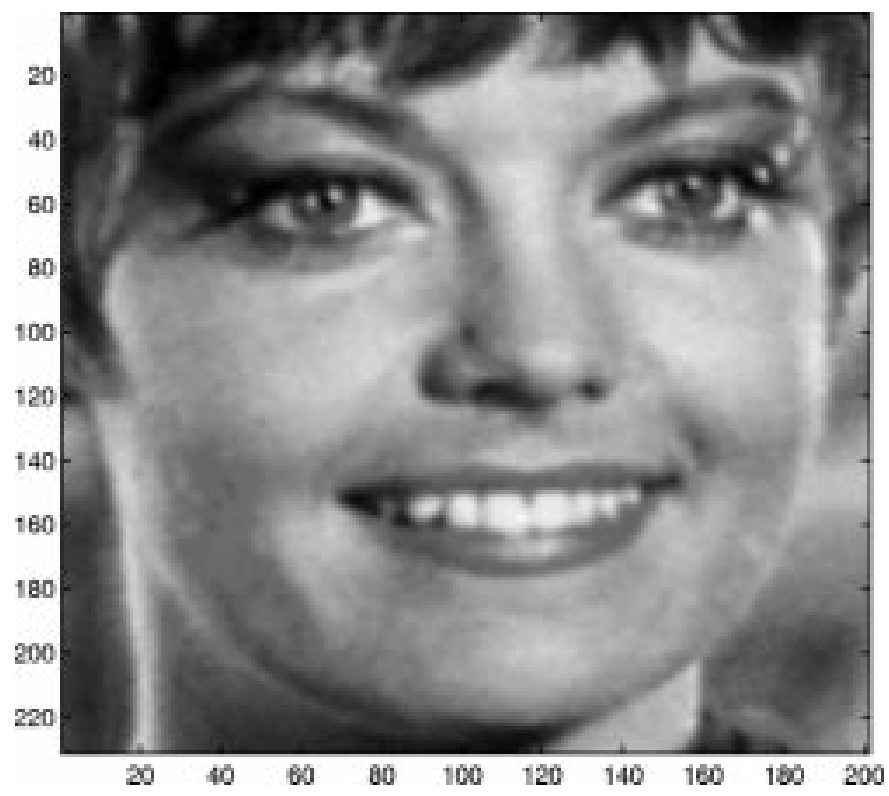

(b)

Fig. 5. (a) Zelda image error-diffused to 2 bpp. (b) Restored Zelda image $(\mathrm{PSNR}=35.39 \mathrm{~dB})$

Peppers image in [4] and let $x_{e}$ be the resulting image after applying our method. The image $x_{e}$ is obtained after a single set of iterations, i.e., $x_{e}=\left(P_{1,1} \circ P_{1,2} \circ \ldots P_{1, L}\right) x_{w}$. The resulting improvement is about $0.5 \mathrm{~dB}$ over the image $x_{w}$. The image $x_{e}$ is shown in Fig. 6 which has a PSNR $=30.90 \mathrm{~dB}$.

Comparison of the POCS-based method with other existing methods are given in Table II for the Peppers and $512 \times 512$ Lena images. Our method results in a higher PSNR than the other two methodsin [1], [4] for both of theimages. In [6], a PSNR of 32.41 is reported for a $200 \times 200$ region containing Lena's face. In the same region, we get a PSNR of 32.51. In order to reconstruct this image, we start with wavelet-based space-scale domain projection. After this, we perform four cycles of orthogonal and nonorthogonal projections onto the sets $C_{1, n}$ with $\lambda=1,0.75,0.5$, and 0.25 . We low-pass filter the resulting image and then perform another two 


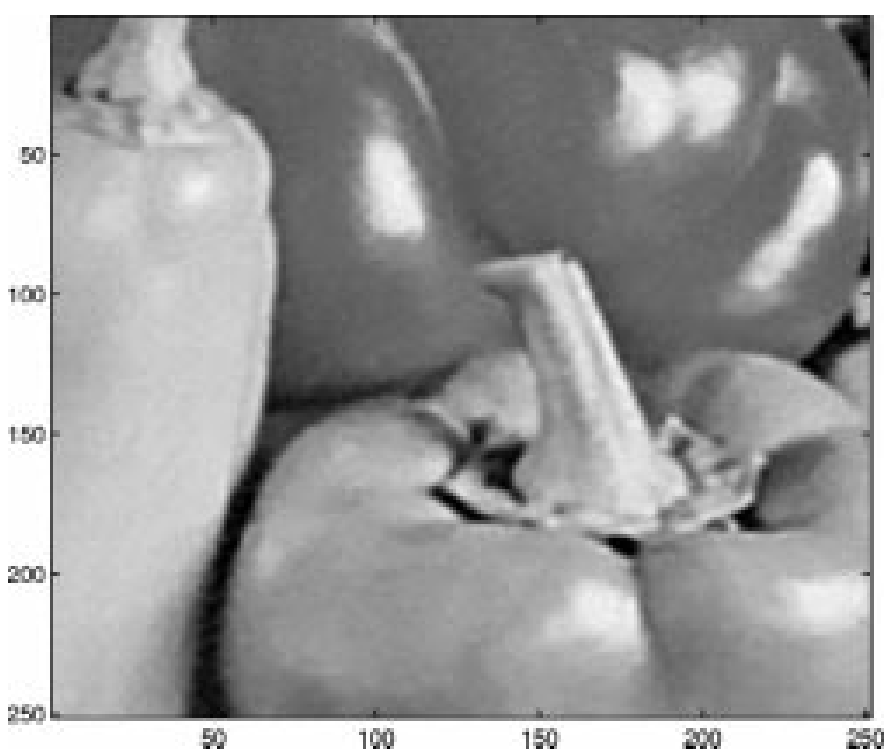

Fig. 6. Restored peppers image $(\mathrm{PSNR}=30.90 \mathrm{~dB})$.

TABLE II

Comparison of InVERSE Half- TONING Methods. All Methods Assume THE ERROR DIFFUSION KERNEL IS KNOWN

\begin{tabular}{c|c|c|c}
\hline & Method[1] & Method [4] & Our Method \\
\hline \hline Lena (PSNR) & 32.00 & 31.67 & 32.18 \\
\hline Peppers (PSNR) & 30.30 & 30.69 & 30.90 \\
\hline
\end{tabular}

cycles of orthogonal and nonorthogonal projections onto the sets $C_{1, n}$ with $\lambda=1$ and 0.5 .

Sample simulation results of the work presented can be viewed at http://www4.ncsu.edu/ gbozkur/invhalftone.html.

\section{REFERENCES}

[1] P. W. Wong, "Inverse half-toning and kernel estimation for error diffusion," IEEE Trans. Image Processing, vol. 4, pp. 486-498, Apr. 1995.

[2] S. Hein and A. Zakhor, "Half-tone to continuous-tone conversion of error-diffusion coded images," IEEE Trans. Image Processing, vol. 4, pp. 208-215, Feb. 1995.

[3] R. L. Stevenson, "Inverse half-toning via map estimation," IEEE Trans. Image Processing, vol. 6, pp. 574-583, Apr. 1997.

[4] Z. Xiong, M. T. Orchard, and K. Ramchandran, "Inverse half-toning using wavelets," in Proc. IEEE Int. Conf. Image Processing, 1996, pp. 569-572.
[5] M. Analoui and J. Allebach, "New results on reconstruction of continuous-tone from half-tone," in Proc. IEEE Int. Conf. Acoust., Speech, Signal Processing, 1992, pp. 313-316.

[6] N. T. Thao, "Set theoretic inverse half-toning," in Proc. IEEE Int. Conf. Image Processing, 1997, pp. 783-786.

[7] S. Mallat and S. Zhong, "Characterization of signals from multiscale edges," IEEE Trans. Pattern Anal. Machine Intell., vol. 14, pp. 710-732, July 1992.

[8] A. E. Çetin and R. Ansari, "Signal recovery from wavelet transform maxima," IEEE Trans. Signal Processing, vol. 42, pp. 194-196, Jan. 1994.

[9] A. H. Tewfik and H. Zou, "Completeness of arbitrarily sampled discrete time wavelet transforms," IEEE Trans. Signal Processing, vol. 43, pp. 2570-2581, Nov. 1995

[10] G. Bozkurt, "Novel methods in image half-toning," M.S. thesis, Bilkent Univ., Ankara, Turkey, 1998.

[11] P. L. Combettes, "The foundations of set theoretic estimation," Proc. IEEE, vol. 81, pp. 182-208, Feb. 1993.

[12] D. C. Youla and H. Webb, "Image restoration by the method of convex projections: Part 1-Theory," IEEE Trans. Med. Imag., vol. MI-1, no. 2, pp. 81-94, 1982.

[13] M. I. Sezan and H. Stark, "Image restoration by the method of convex projections: Part II-Applications and numerical results," IEEE Trans. Med. Imag., vol. MI-1, no. 2, pp. 95-101, 1982.

[14] J. S. Lim, Two-Dimensional Signal and Image Processing. Englewood Cliffs, NJ: Prentice-Hall, 1990.

Gozde Bozkurt Unal (S'00) received the B.Sc. degree in electrical engineering from Middle East Technical University, Ankara, Turkey, in 1996, and the M.Sc. degree in electrical engineering from Bilkent University, Ankara, in 1998. She is currently a Research Assistant in the Electrical and Computer Engineering Department at North Carolina State University, Raleigh, where she is pursuing the Ph.D. degree.

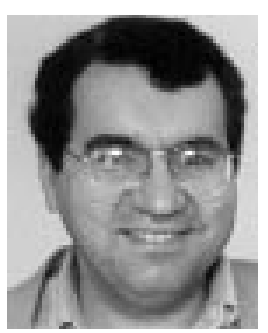

A. Enis Çetin (S'85-M'87-SM'95) received the B.Sc. degree in electrical engineering from Middle East Technical University, Ankara, Turkey, and the M.S.E., and Ph.D. degrees in systems engineering from the Moore School of Electrical Engineering at the University of Pennsylvania, Philadelphia.

From 1987 to 1989, he was Assistant Professor of Electrical Engineering at the University of Toronto, Toronto, ON, Canada. Since then, he has been with Bilkent University, Ankara, where he is currently a Full Professor. During the summers of 1988, 1991, and 1992, he was with Bell Communications Research (Bellcore). He spent the 1996-1997 academic year at the University of Minnesota, Minneapolis, as a Visiting Associate Professor. Currently, he is with Sabanci University, Istanbul Turkey.

Dr. Çetin is a Member of the DSP technical committee of the IEEE Circuits and Systems Society and an Associate Editor of IEEE TRANSACTIONS ON IMAGE PROCESSING. He founded the Turkish Chapter of the IEEE Signal Processing Society in 1991. He received the Young Scientist Award of TUBITAK (Turkish Scientific and Technical Research Council) in 1993. He was the Chair of the IEEE-EURASIP Nonlinear Signal and Image Processing Workshop (NSIP'99) held in Antalya, Turkey, in June 1999. 